\title{
Auswirkungen der Peer-Review-Verfahren: Veränderungen der Fehlerkultur und der internen Abläufe in den Fachgruppen und Kliniken
}

\section{J. Zacher}

Qualitätsmanagement

Schlüssselvörter

medizinische Qualität

Fehlerkultur

Qualitätsmanagement

Key words

medical quality

failure management

quality management
Institut

HELIOS Klinikum Berlin Buch

Bibliografie

DOI $10.1055 / \mathrm{s}-0028-1085592$

Dtsch Med Wochenschr 2008;

133: S148 - (c) Georg Thieme

Verlag KG Stuttgart - New York

ISSN 0012-0472

\section{Korrespondenz}

\section{Prof. Dr. med. Josef Zacher,} HELIOS Klinikum Berlin Buch Schwanebecker Chaussee 50 13125 Berlin

Tel. +49 (30) 940152301

eMail josef.zacher@

helios-kliniken.de
Medizinische Qualität wird in Zertifizierungsverfahren meist über Struktur und Prozesse definiert. In den HELIOS-Kliniken steht unter dem Motto „Ergebnis zählt“ die medizinische Ergebnisqualität, generiert aus Routinedaten, im Vordergrund. Problematisch bleibt in allen diesen Verfahren die Umsetzung der formulierten Ziele in den klinischen Alltag. Auch in den HELIOS-Kliniken existierte und existiert dieses Problem.

\section{Strukturen zur Umsetzung medizinischer Qualität}

Die HELIOS-Kliniken haben im letzten Jahrzehnt Strukturen zur Umsetzung definierter Qualitätsziele entwickelt. Alle Chefärzte, z.B. Orthopäden/ Unfallchirurgen, einer Fachrichtung bilden eine Fachgruppe. Die Abteilung Medizinische Entwicklung vereinbart zusammen mit der Fachgruppe medizinische Ergebnisziele (z.B. Mortalität nach elektiver Hüftendoprothese). Diese orientieren sich an nationalen und internationalen Kennzahlen. Diese Ergebnisqualität wird an alle Chefärzte der HELIOS-Kliniken regelmäßig kommuniziert. Numerisch auffällige, weil vom vereinbarten Ziel abweichende, Kliniken werden einem Peer-Review-Verfahren zugeführt. Dabei werden die Akten von definierten Indexpatienten (z.B. Verstorbene nach elektiver Hüft-TEP) von erfahrenen HELIOS-Chefärzten unterschiedlicher Fachdisziplinen auf Verbesserungspotential überprüft. Die Ergebnisse dieser Reviews werden intern im Detail, extern in Summe publiziert. Als wesentliches Ergebnis stellt sich meist heraus, dass Verbesserungspotential in der interdisziplinären Zusammenarbeit gesehen wird.

\section{Veränderung in Fachgruppen und in den Kliniken}

Das Messen von medizinischen Ergebnissen und das offene Umgehen mit guten wie schlechten Ergebnisdaten ist auch für TOP-Mediziner eine neue und nicht immer angenehme Erfahrung. Angst vor Sanktionen und vor unangenehmen Nebenwirkungen dieser publizierten Ergebnisse bestimmten anfangs die Diskussionskultur. Die Erkenntnis der Chefärzte, dass das Verbesserungspotential bzw. Fehler nicht unbedingt in einer einzelnen Abteilung, wohl aber in der Verbesserung der interdisziplinären Patientenbetreuung liegt, führt dazu, dass vor allem bei den untersuchten Tracerdiagnosen (z.B. elektive Hüft-TEP), letztlich aber auch insgesamt in den

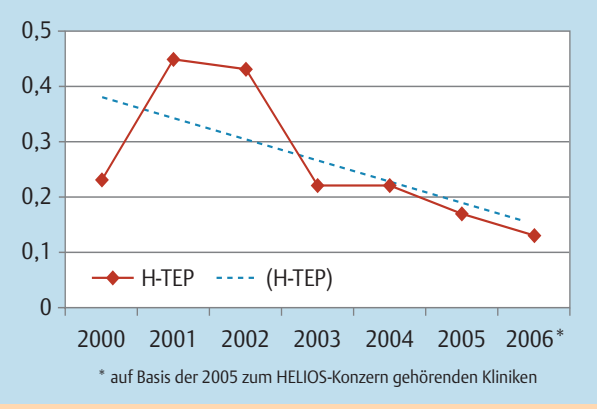

Abb. 1 Krankenhausmortalität bei elektiver Hüft-TEP.

Abteilungen Wege erarbeitet werden, wie die erkannten Problemkreise durch neue Verfahrensanweisungen positiv beeinflusst werden können. Innerhalb der einzelnen Kliniken wie auch zwischen den Fachgruppen findet ein gesteuerter Austausch über diese Optimierungsmöglichkeiten statt.

\section{Veränderung von medizinischen Ergebnissen}

In einer Vielzahl von untersuchten Fällen konnte die medizinische Ergebnisqualität im Laufe der überprüften Jahre verbessert werden (Abb. 1). Dies ist natürlich nicht allein auf das Peer-Review-Verfahren in wenigen Kliniken zurückzuführen, sondern liegt in der gewachsenen Erkenntnis aller Kliniken begründet, dass medizinische Qualität messbar sowie verbesserbar ist. Regelmäßiges offenes Reporting von medizinischen Ergebnisdaten, detailliertes Hinsehen in Abteilungen, die von dieser Qualität abweichen, konstruktive externe Hilfe bei erkannten Problemen in Strukturen und Prozessen sind der Weg, an einer kontinuierlichen Verbesserung bzw. dem Erhalt der medizinischen Qualität zu arbeiten. Da im Sinne der Entropie eine hohe Tendenz zum Qualitätsverlust besteht, ist es eine kontinuierliche Aufgabe, an diesen Zielen und deren Verbesserung zu arbeiten.

Autorenerklärung: Es bestehen keine finanziellen Interessenkonflikte in Zusammenhang mit diesem Artikel. 